\title{
Collision experiments with Stark-decelerated beams
}

\author{
Sebastiaan Y. T. van de Meerakker* and Gerard Meijer
}

\author{
Received 5th November 2008, Accepted 16th December 2008 \\ First published as an Advance Article on the web 29th April 2009 \\ DOI: $10.1039 / b 819721 k$
}

\begin{abstract}
The crossed molecular beam technique has been established as a mature and important experimental method for detailed studies of molecular interactions, and has contributed enormously to our present understanding of molecular reaction dynamics. The Stark deceleration technique yields unprecedented control over both the internal and external degrees of freedom of polar molecules in a molecular beam, offering new possibilities in scattering experiments. In particular, Stark-decelerated molecular beams allow detailed molecular scattering studies as a function of the collision energy, from low to high collision energies, and with a very high energy resolution. We discuss a variety of experimental geometries that exploit this new molecular beam technology for scattering experiments, ranging from crossed beam arrangements and molecular synchrotrons to surface scattering set-ups.
\end{abstract}

\section{Introduction}

Acquiring a detailed understanding of the interaction between individual molecules is a central theme in molecular physics, and is essential, for instance, to understand the dynamic behavior of larger systems. The study of collisions between neutral atoms and molecules in the gas-phase is a well-established approach to probe the potential energy surfaces (PES) that govern molecular interactions. The level of detail that can be reached in these experiments depends on the quality of the preparation of the collision partners prior to the collision event. "In the ultimate experiment one would determine the cross section and angular distribution of the products for a completely specified collision", as Raphael Levine and Richard Bernstein put it in their classic book "Molecular Reaction Dynamics and Chemical Reactivity". ${ }^{1}$ The parameters that are to be specified include the quantum state of the reactants and products, the alignment or orientation of the reactants and products, the angular and velocity distribution of the products, and the energy of the collision. Experimentally, the crossed molecular beam technique is ideally suited to obtain detailed information of the PES. ${ }^{2}$ Crossed molecular beams enable the study of molecular encounters under single collision conditions, and allow the realization of many of the desiderata listed above. ${ }^{3}$ A rich variety of experimental geometries have been engineered to obtain control over the internal quantum states, ${ }^{4}$ the orientation, ${ }^{5}$ and the collision energy of the scatterers. ${ }^{6,7}$ Sophisticated laser-based detection methods have been developed to analyze the state, angular, and velocity distributions of the molecules after the collision. ${ }^{8-11}$ These techniques also allowed the determination of product pair correlations in bi-molecular reactions. ${ }^{12}$

Stark-decelerated molecular beams offer interesting new prospects for increased control over the molecular reactants in scattering experiments. The Stark decelerator

Fritz-Haber-Institut der Max-Planck-Gesellschaft, Faradayweg 4-6, 14195 Berlin, Germany. E-mail: basvdm@fhi-berlin.mpg.de 
for neutral polar molecules is the equivalent of a linear accelerator (LINAC) for charged particles, and exploits the interaction of a polar molecule with inhomogeneous time-varying electric fields. ${ }^{13}$ The deceleration (or acceleration) process can be seen as slicing a packet of molecules with a narrow velocity distribution out of the most intense part of the molecular beam pulse. This packet can then be decelerated or accelerated to any velocity, maintaining the narrow velocity distribution and the particle density in the packet. In a crossed beam configuration, this offers the revolutionary possibility to study fully state-selected (in)elastic and reactive scattering as a function of the continuously variable collision energy with a high intrinsic energy resolution. The computer-controlled velocity of the molecular beam(s) allows one to scan the collision energy in an otherwise fixed experimental geometry. The deceleration process is highly quantum-state specific, and the state purity of the bunches of selected molecules that emerge from the decelerator is measured to be close to $100 \%$. Contamination of inelastic state-to-state scattering data by initial populations in different quantum states is therefore negligible. The high state purity of the beam also allows sensitive background-free detection of inelastically scattered products. The molecules that exit the decelerator are all naturally spatially oriented, allowing steric effects to be studied. Last but not least, the possibility to produce molecular beams with a low velocity gives access to scattering studies at low collision energies, an experimentally almost unexplored regime.

One of the interesting aspects of cold collisions involving molecules is the occurrence of scattering resonances. These resonances occur, for instance, when the collision energy is degenerate with a bound state of the collision complex. ${ }^{14}$ These resonant states can also be formed near the energetic thresholds for inelastic scattering, where the collision deposits all available energy into internal molecular degrees of freedom. ${ }^{15}$ Molecular collision studies as a function of the collision energy, and the careful mapping of scattering resonances, provide sensitive probes for the potential energy surfaces. Low collision energies also allow external control over the collision dynamics by electromagnetic fields. ${ }^{16}$ At collision energies near one Kelvin, the perturbations due to the Zeeman and/or Stark effect become comparable to the translational energy. In this regime, molecular collisions are highly susceptible to externally applied fields, opening the possibility for controlled chemistry. ${ }^{17}$

These "tamed" molecular beams that have been produced using either electric, magnetic or optical fields, ${ }^{18}$ can be used to advantage in a new generation of scattering experiments. It is the challenge, however, to design experiments that exploit these new possibilities while maintaining the already existing high level of control that has set the standard in the field of molecular scattering. In this paper we will present our view of how the Stark deceleration technique can be implemented in scattering experiments to probe the intermolecular interaction potential in detail. We will discuss the feasibility of these experiments, and we will describe the first bi-molecular study that is planned in our laboratory, i.e., the scattering between Stark-decelerated beams of fully state-selected $\mathrm{OH}\left(X^{2} \Pi_{3 / 2}, J=3 / 2, f\right)$ and $\mathrm{NO}$ $\left(X^{2} \Pi_{1 / 2}, J=1 / 2, f\right)$ radicals. Finally, we will discuss the potential of molecular storage rings for scattering experiments and we will describe a surface scattering experiment with slow, ground-state $\mathrm{CO}$ molecules that is currently being set up.

\section{The density question}

A critical parameter that determines the success of any crossed beam scattering experiment is the product of the molecular particle densities ${ }^{50}$ of the colliding beams. The particle densities in the colliding beams should be sufficient to build up a detectable signal of the scattering products in the time the two beams intersect. Unfortunately, the relevant state-to-state inelastic collision rates for bi-molecular scattering are not accurately known and vary from system to system, but we can estimate the required particle densities by assuming a scattering rate $k$ of $10^{-10}-10^{-11} \mathrm{~cm}^{3}$ molecule $^{-1} \mathrm{~s}^{-1}$. These rates are typically found for diatom-rare gas atom systems at 
high collision energies, as well as for collisions between di-atoms at low collision energies. ${ }^{19}$ The required particle densities $n_{\mathrm{A}}$ and $n_{\mathrm{B}}$ in each of the beams can now be estimated from

$$
N_{\text {product }}=n_{\mathrm{A}} n_{\mathrm{B}} k V \Delta t
$$

where $N_{\text {product }}$ is the number of scattered molecules just after the beams have collided, and $\Delta t$ is the time during which both beams interact. The scattering volume $V$ in our experiments is approximately $3 \times 3 \times 3 \mathrm{~mm}^{3}$. The detection limit of the scattering products depends strongly on the molecular species of interest, on the detection technique that is used, on the spatial overlap of the detection laser with the scattering volume as well as on the background level in the experiment. In pulsed beam experiments, product molecular densities $\left(N_{\text {product }} / V\right)$ typically between $10^{3}-10^{4}$ $\mathrm{cm}^{-3}$ can be detected with a reasonable signal-to-noise ratio within a reasonable signal accumulation time. We will use this number density as a figure of merit to estimate the required particle densities in the colliding beams, and realize that for selected systems this estimate is under- or over-valued.

We assume that detectable scattering products are only produced during an interaction time of $\sim 10 \mu \mathrm{s}$ of the intersecting beams, leading to a required product density $n_{\mathrm{A}} n_{\mathrm{B}}$ of $10^{18}-10^{20} \mathrm{~cm}^{-6}$ to be able to experimentally observe scattering. Note that the particle density in each individual beam is in principle inconsequential, as the state purity of both beams is close to $100 \%$. The inelastically scattered molecules can therefore be detected background free, independent of the particle density and rotational temperature of the parent molecular beams.

\section{Molecular traps}

It appears appealing to relax the requirements on the particle densities by increasing the interaction time between the molecules. In recent years a variety of traps, either electric, ${ }^{20,21}$ magnetic, or a combination of both, ${ }^{22}$ have been developed that allow the confinement of molecules for times up to several seconds. ${ }^{23}$ Traps offer an interaction time that is typically five orders of magnitude larger than the interaction time in a crossed beam configuration. This advantage is deceptive, however, as the scattered products are, in general, not accumulated during this interaction time. In most trapping experiments, molecules are initially confined in a low-field seeking quantum state. If the collision induces a transition from the low-field seeking into a high-field seeking state, the molecules can no longer be confined to the trap. Inelastic channels are usually also available that populate low-field seeking states that can potentially be confined to traps. However, these collisions either populate hyperfine states that cannot be probed independently from the population in the initial quantum state, or provide the scattered molecules with a recoil energy that is larger than - or comparable to - the trap depth. Scattering between molecules in a trap can therefore only be inferred from the observed depletion of trapped molecules as a function of time. To be able to experimentally identify trap loss due to collisions of trapped molecules, this density dependent loss channel needs to be comparable in magnitude to the other loss mechanisms. Referring back to eqn 1 , even a molecular density of $10^{9}$ molecules $\mathrm{cm}^{-3}$ in the trap will only lead to a $10 \%$ reduction of the trapped molecules in the first second of trapping, if we assume a scattering rate of $10^{-10} \mathrm{~cm}^{3}$ molecule $\mathrm{s}^{-1}$. Clearly, the advantage of the long interaction time afforded by the trap is undone by the inferior detection sensitivity of the scattering events.

Detailed molecular scattering studies are therefore better performed in a beam environment than in a trap. The study of molecular collisions in the cold $(1 \mathrm{mK}-1$ $\mathrm{K})$ temperature regime, i.e., at temperatures that are routinely reached in traps, is also possible with molecular beams when two merging molecular beams with a well-defined velocity are used that slowly overtake each other. This experimental arrangement has the advantage that the collision energy can be varied, and that 
experimental parameters in the interaction region can easily be controlled. Moreover, the molecular densities that are reached in decelerated beams are generally higher than those that are obtained in traps. In section VII we discuss different experimental possibilities to achieve merged molecular beams.

The confinement of molecules in traps is required to study molecular collisions in the ultracold $(<1 \mathrm{mK})$ regime that is inaccessible to crossed beam configurations. These collisions are dominated by $s$ or $p$-wave scattering and are sensitive to the long-range part of the potential. In particular the dipole-dipole interaction for polar species gives rise to rich physics that is foreign to collisions at higher temperatures. ${ }^{24,25}$ Recently, tremendous progress has been made in producing ground-state ultracold polar molecules via photoassociation spectroscopy, ${ }^{26-28}$ bringing ultracold bi-molecular collision studies of alkali di-atoms within reach. For more chemically relevant molecules like ammonia or the hydroxyl radical that have only been trapped at tens of $\mathrm{mKs}$ thus far, further cooling schemes like evaporative or sympathetic cooling need to be developed.

\section{Scattering Stark-decelerated $\mathrm{OH}$ radicals with Xe atoms}

Recently, we have carried out the first crossed beam scattering experiment using a Stark-decelerated beam. In this experiment, a Stark-decelerated beam of $\mathrm{OH}$ radicals collided with a conventional beam of Xe atoms. ${ }^{29}$ The velocity tunability and the narrow velocity spread of the $\mathrm{OH}$ beam allowed us to tune the center-of-mass collision energy over the energetic thresholds for inelastic scattering into rotationally excited states. The velocity of the incoming $\mathrm{OH}$ radicals was varied from $33 \mathrm{~m} \mathrm{~s}^{-1}$ to $700 \mathrm{~m} \mathrm{~s}^{-1}$, and the behavior of the cross sections for inelastic scattering around the energetic thresholds were accurately measured. In Fig. 1 the measured relative inelastic scattering cross sections are shown as a function of the collision energy for the scattering channels that are indicated in the figure.

A new ab initio potential energy surface for the $\mathrm{OH}-\mathrm{Xe}$ system was calculated, and fully converged coupled-channels calculations were performed. The resulting

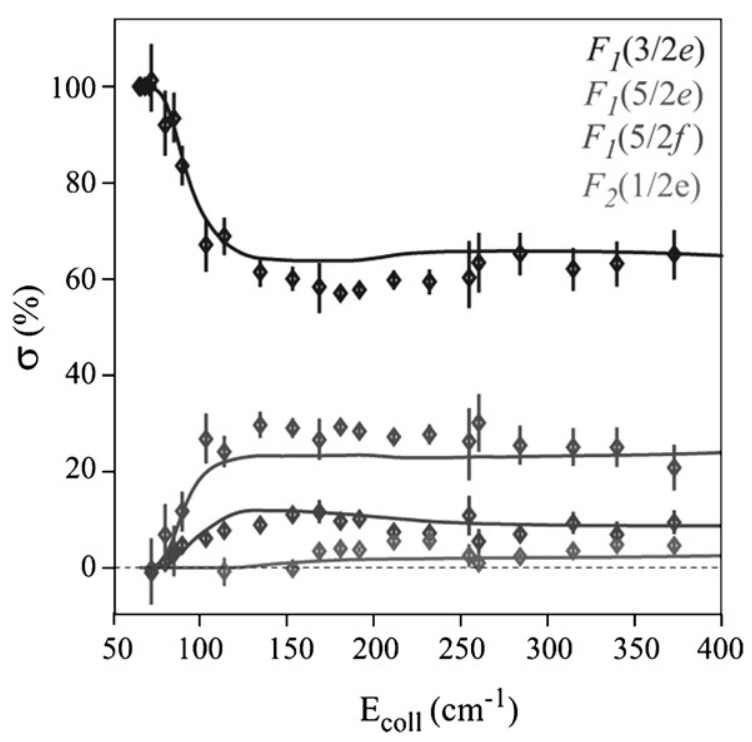

Fig. 1 Collision energy dependence of the relative inelastic collision cross section for scattering of $\mathrm{OH}\left(X^{2} \Pi_{3 / 2}, J=3 / 2, f\right)$ radicals with $\mathrm{Xe}$ atoms for the indicated scattering channels (see Fig. 3 for a rotational energy level diagram of the $\mathrm{OH}$ radical). The solid lines result from ab initio coupled-channels calculations. 
relative inelastic cross-sections are shown as solid lines in Fig. 1 and agree excellently with the measured inelastic cross-sections throughout the range of collision energies that were probed.

In the $\mathrm{OH}-\mathrm{Xe}$ experiment, the energy resolution of about $13 \mathrm{~cm}^{-1}$ that could be obtained was almost exclusively determined by the velocity spread in the Xe beam. The logical next step is to develop experimental methods to study bi-molecular collisions using two decelerated molecular beams under a 90 degree crossing angle. In such an experiment, molecular scattering between (combinations of) fully state-selected molecules like $\mathrm{OH}, \mathrm{NH}, \mathrm{ND}_{3}, \mathrm{CO}, \mathrm{H}_{2} \mathrm{CO}$, and $\mathrm{SO}_{2}$ can be studied with a collision energy that can be varied from below $1 \mathrm{~cm}^{-1}$ to conventional collision energies (few hundred $\mathrm{cm}^{-1}$ ). The energy resolution is $\leq 1 \mathrm{~cm}^{-1}$, one order of magnitude better than in the $\mathrm{OH}-\mathrm{Xe}$ experiment, and typically two orders of magnitude better than in conventional crossed molecular beam experiments. In these experiments, detailed studies of threshold phenomena, scattering resonances, and collisions at low temperatures are possible.

\section{More efficient Stark decelerators}

The particle densities that have been obtained thus far in Stark-decelerated molecular beams are, unfortunately, insufficient for scattering studies using two crossed Stark-decelerated beams. Depending on the molecular species and the final velocity of the packet of molecules, densities that are typically reached range from $10^{6}$ to $10^{9}$ molecules $\mathrm{cm}^{-3}$. From Stark deceleration and trapping experiments in our laboratory, we know that the highest densities can be obtained for the molecules $\mathrm{ND}_{3}$ and $\mathrm{CO}\left(a^{3} \Pi\right)$. The density of decelerated $\mathrm{OH}$ beams, although often used in deceleration and trapping experiments, is already one to two orders of magnitude less, while decelerated beams of $\mathrm{NH}\left(a^{1} \Delta\right)$ radicals are yet somewhat less intense.

During the last few years, an improved understanding of the motion of molecules in a Stark decelerator has been obtained. In particular, it was found that the

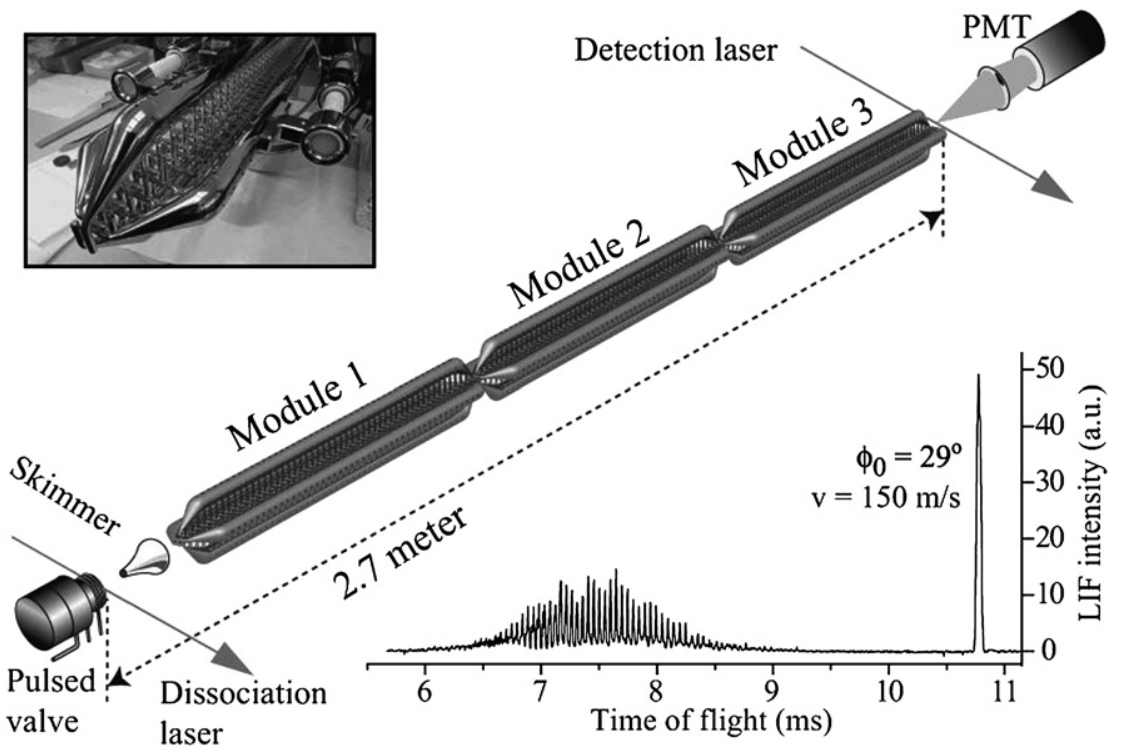

Fig. 2 A 2.6 meter long Stark decelerator that consists of 3 modules of about 100 electric field stages each. In the top left inset a photograph of a decelerator module is shown. In the bottom right corner an experimental time of flight profile of $\mathrm{OH}$ radicals is shown that is recorded at the exit of the decelerator. In this measurement, a packet of $\mathrm{OH}$ radicals is decelerated from $350 \mathrm{~m}$ $\mathrm{s}^{-1}$ to $150 \mathrm{~m} \mathrm{~s}^{-1}$, using the decelerator in the $s=3$ overtone mode at a phase angle of $29^{\circ}$. 
coupling between the longitudinal and transverse motion of the molecules can substantially reduce the number of molecules that reach the exit of the decelerator. ${ }^{30}$ In a Stark decelerator with the original electrode geometry, ${ }^{13}$ this coupling between the longitudinal and transverse motion can be effectively switched off when the decelerator is operated in the so-called $s=3$ overtone mode. ${ }^{31}$ In this mode, only every third electric field stage is used for deceleration, while extra transverse focusing is provided by the intermediate stages. The performance of this scheme has been experimentally studied in our laboratory by passing a beam of $\mathrm{OH}$ radicals through a 2.6 meter long Stark decelerator consisting of 316 electric field stages. ${ }^{32}$ A schematic representation of this Stark decelerator is shown in Fig. 2, together with a typical measured Time Of Flight (TOF) profile of a beam of $\mathrm{OH}$ radicals that exits the decelerator.

In this machine, the $\mathrm{OH}$ radicals can be detected either after 103 or after 316 electric field stages, enabling a direct comparison between the conventionally used mode of operation $(s=1)$ and the $s=3$ overtone mode of operation under otherwise identical conditions, in particular using the same phase angle $\phi_{0}$. We have demonstrated and quantified that indeed for many applications, the acceptance of the Stark decelerator in the $s=3$ mode significantly exceeds that of a decelerator in the conventionally used mode of operation. A gain up to a factor of 4 is observed, and is found to depend strongly on the phase angle that is used. Simulations indicate that, compared to the decelerators that have been commonly used so far $\left(s=1, \approx 100\right.$ stages, $\phi_{0}=$ $50-60^{\circ}$ ), a gain of a factor of 10 is realistic for a large range of final velocities if the decelerator were to be built even longer. It is important to note that this gain in number density is accompanied by a reduction in the longitudinal translational temperature by a factor of $3 .^{31}$ With this new deceleration scheme, scattering experiments between Stark-decelerated molecular beams have become feasible.

\section{Scattering between $\mathrm{OH}$ and NO radicals}

Based on scientific relevance and feasibility, the first bi-molecular scattering experiment that is planned in our laboratory using two crossed Stark decelerators is the scattering between state-selected $\mathrm{OH}\left(X^{2} \Pi_{3 / 2}, J=3 / 2\right)$ and $\mathrm{NO}\left(X^{2} \Pi_{1 / 2}, J=1 / 2\right)$ radicals. Collisions involving radicals, and in particular the $\mathrm{OH}$ and the NO radical, play a key role in atmospheric and interstellar chemistry as well as in combustion processes. ${ }^{33}$ Hence, the scattering of $\mathrm{OH}$ and $\mathrm{NO}$ with (mostly) rare-gas atoms has received much experimental and theoretical attention. For both species, ingenious experiments have been performed in which the radicals have been stateselected, ${ }^{34,35}$ and in which steric effects have been studied by orienting the radicals prior to the collision by static electric fields. ${ }^{36,37}$ Recently, reactive scattering has also been observed using state-selected beams of $\mathrm{OH}$ radicals. ${ }^{38}$ Theoretically, high level ab initio PES's have been calculated, and the comparison with experimental data has yielded a wealth of information on the interaction between open shell ${ }^{2} \Pi$ molecules and other atoms or molecules.

As a prototypical system for the interaction between $t w o$ open shell ${ }^{2} \Pi$ molecules, we plan to study the inelastic scattering between fully state-selected $\mathrm{OH}$ and $\mathrm{NO}$ molecules as a function of the collision energy. This will provide detailed information on the intermolecular interactions in this system, ${ }^{39}$ and will probe the potential energy surface beyond the bound part of the potential that can be studied spectroscopically. ${ }^{40}$ Although challenging, these systems are also still theoretically tractable, ${ }^{41}$ so that experimental cross sections can be compared to theoretically calculated ones. These studies are not only relevant for atmospheric and combustion processes but are also relevant to predict the feasibility of evaporative cooling of molecules like $\mathrm{OH}$ in electric or magnetic traps, that solely relies on the collision properties of ${ }^{2} \Pi$ molecules at low collision energies.

The rotational energy level schemes of the $\mathrm{OH}$ and $\mathrm{NO}$ radicals are shown in Fig. 3. The spin-orbit interaction splits the $X^{2} \Pi$ electronic state into two rotational 


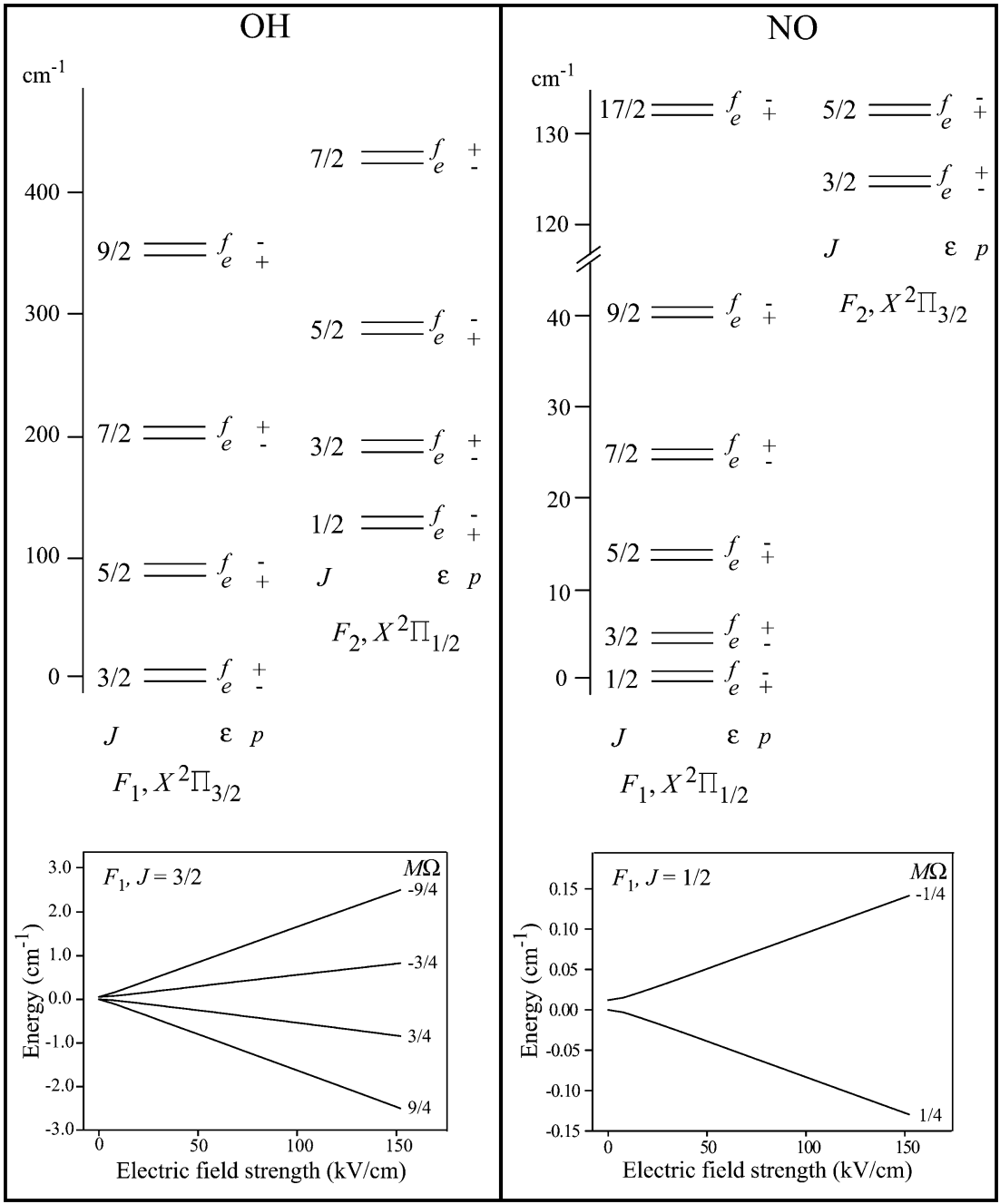

Fig. 3 Rotational energy level diagram of the electronic and vibrational ground states of the $\mathrm{OH}$ (left) and NO (right) radicals. The $\Lambda$-doublet splitting of the energy levels is greatly exaggerated for reasons of clarity. The parity and spectroscopic symmetry labels are indicated for each level. The Stark energy diagram for the rotational ground state is shown in the lower part for both species. Note the different energy scales that are used.

manifolds with $|\Omega|=1 / 2$ and $|\Omega|=3 / 2$, respectively. In the united atom picture, the NO radical has one electron in the $3 \mathrm{p} \pi$ valence shell, and the $|\Omega|=1 / 2$ manifold is lower in energy than the $|\Omega|=3 / 2$ manifold. The $\mathrm{OH}$ radical has three electrons in the $2 \mathrm{p} \pi$ shell, one electron short of filling the shell. Consequently, the energy level scheme of the $\mathrm{OH}$ radical is said to be inverted and the $|\Omega|=3 / 2$ manifold is lower in energy than the $|\Omega|=1 / 2$ one. The $\mathrm{OH}$ and NO radicals have rotational constants of 18.5 and $1.7 \mathrm{~cm}^{-1}$, respectively, and the rotational energy level spacing is thus much larger for $\mathrm{OH}$ than for NO. Both molecules have a spin-orbit constant that is similar in magnitude $\left(-139.7 \mathrm{~cm}^{-1}\right.$ for $\mathrm{OH}$ and $123.1 \mathrm{~cm}^{-1}$ for $\left.\mathrm{NO}\right)$.

A schematic representation of the crossed beam scattering machine that is being developed in our laboratory is shown in Fig. 4. The machine consists of two Stark decelerators under a 90 degree crossing angle, where each decelerator consists in 


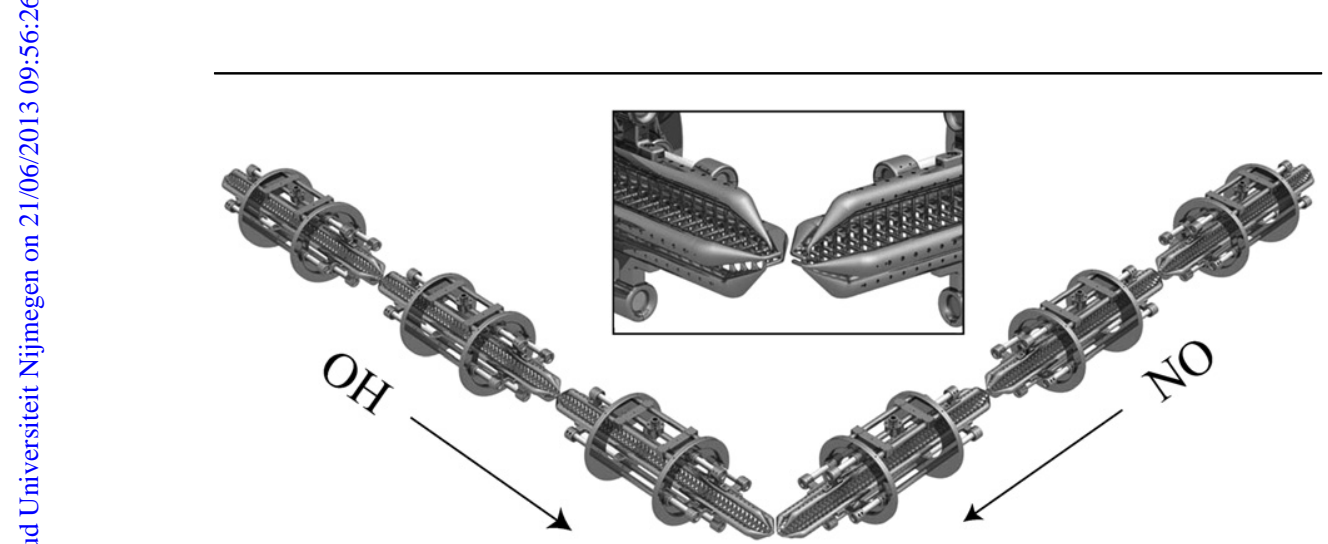

Fig. 4 Schematic representation of the crossed beam machine. Each decelerator has a modular design and consists in total of 316 electric field stages. The decelerators are designed such that the last electric field stages of either one of the decelerators are very close to the collision zone, while at the same time providing excellent optical access to detect the scattering products (Design: Henrik Haak).

total of 316 electric field stages and has a length of 2.6 meters. Both decelerators have the same design. The last electric field stages are mounted on conically shaped rods. This allows the exits of both decelerators to be very close to the collision zone, while providing excellent optical access to detect the scattering products. In the crossed beam experiment with $\mathrm{OH}$ and $\mathrm{NO}$ radicals, one decelerator will be used to accelerate or decelerate a beam of $\mathrm{OH}$ radicals as has been described in section $\mathrm{V}$. The second decelerator will be used to produce cold packets of NO radicals by mere velocity filtering.

The NO radical possesses only a relatively small dipole moment of $0.16 \mathrm{D}$. Therefore, deceleration or acceleration of a beam of $\mathrm{NO}$ molecules in the $X^{2} \Pi_{1 / 2}, J=1 / 2$ state over a large range of velocities is rendered impossible, even for the 300 -stage long decelerators that we are constructing. NO radicals in the $X^{2} \Pi_{3 / 2}, J=3 / 2$ state experience a significantly larger Stark shift, and the deceleration in this state would be possible. This state also possesses a magnetic moment of 1.2 Bohr magneton,

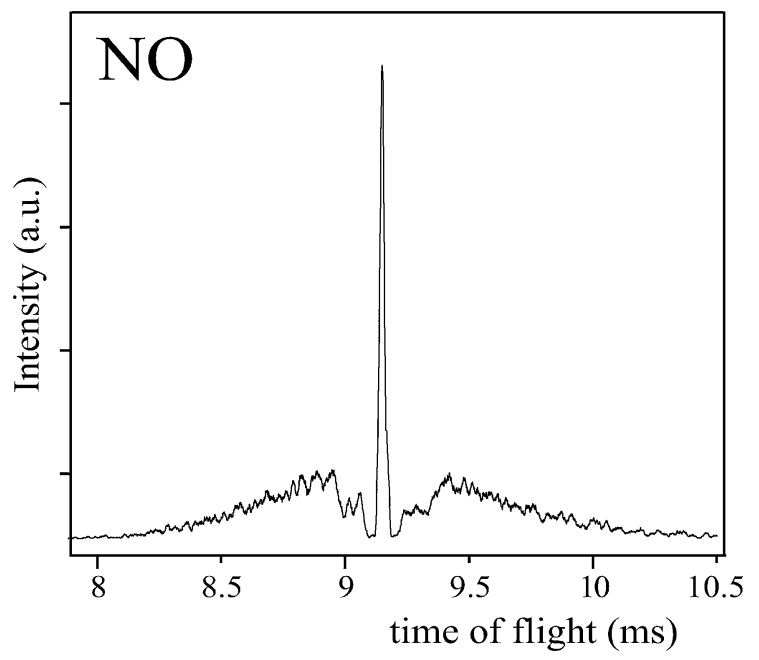

Fig. 5 Simulated time-of-flight profile of a beam of $\mathrm{NO}\left({ }^{2} \Pi_{1 / 2}, J=1 / 2\right)$ radicals that emerges from the 316 stage Stark decelerator when the decelerator is operated at $\phi_{0}=0^{\circ}$. The longitudinal temperature of the molecules in the central narrow peak is about $10 \mathrm{mK}$. 
making it a good candidate for efficient deceleration with a Zeeman decelerator. ${ }^{42}$ Although the $X^{2} \Pi_{3 / 2}, J=3 / 2$ state will hardly be populated in a pulsed molecular beam, NO molecules can be prepared in this state from the rotational ground state by stimulated emission pumping via the $A^{2} \Sigma^{+}$state. The dipole moment of NO molecules is sufficient, however, to guide $\left(\phi_{0}=0^{\circ}\right)^{43} X^{2} \Pi_{1 / 2}, J=1 / 2$ state molecules through the decelerator, leaving the velocity of the selected part of the beam unchanged. In this mode, the decelerator acts as a velocity selector: only molecules within a limited initial velocity range that is determined by the Stark effect of the molecule are kept together, and arrive in the collision region as a compact bunch. The small dipole moment of NO results in a narrow velocity spread and thus a low temperature of the bunch. To produce a cold packet of NO radicals with a velocity that is as low as possible, a cooled pulsed valve will be employed and $\mathrm{Xe}$ will be used as a carrier gas. The TOF profile that can thus be obtained is shown in Fig. 5. In this simulation, a molecular beam of $\mathrm{NO}\left({ }^{2} \Pi_{1 / 2}, J=1 / 2\right)$ molecules with a mean velocity of $280 \mathrm{~m} \mathrm{~s}^{-1}$ and with a $10 \%$ velocity spread is guided through the Stark decelerator. The intense and narrow peak in the center of the arrival time distribution contains the selected part of the beam. These cold NO molecules have a velocity spread of about $3 \mathrm{~m} \mathrm{~s}^{-1}$ (or $1 \%$ of the mean velocity).

The timing of the experiment can be chosen such that both the decelerated/accelerated $\mathrm{OH}$ beam and the cold part of the NO beam arrive simultaneously, but temporally separated from the remainder of the $\mathrm{OH}$ and $\mathrm{NO}$ beam pulses, in the collision zone. The Xe atoms from the carrier gas that is used to produce the NO molecular beam arrive simultaneously with the cold packet of NO radicals in the collision zone. These atoms, however, are not kept together by the Stark decelerator, and their particle density is reduced to a negligible number during the 2.7 meter flight path to the collision center.

When the velocity of the $\mathrm{OH}$ beam is varied between $50 \mathrm{~m} \mathrm{~s}^{-1}$ and $800 \mathrm{~m} \mathrm{~s}^{-1}$, the center-of-mass collision energy is scanned from $35 \mathrm{~cm}^{-1}$ to $325 \mathrm{~cm}^{-1}$. This energy range encompasses many energetic thresholds for inelastic scattering in both the $\mathrm{OH}$ radical and the NO radical, and many resonances in the inelastic cross sections can be expected. The energy resolution is about $1 \mathrm{~cm}^{-1}$ at the lowest collision energies, and scales with the square root of the collision energy.

In view of the small dipole moment of the NO radical, the Stark decelerator will only select a small fraction of the initial pulse of NO radicals. We can estimate this fraction from the calculated acceptance of the Stark decelerator. The acceptance for NO radicals is about one order of magnitude less than the acceptance for $\mathrm{OH}$ radicals under otherwise identical conditions. Molecular beams of NO radicals can be produced with particle densities that exceed the densities that are reached for $\mathrm{OH}$ radicals by orders of magnitude, however. The NO radical is one of the few open shell radicals that is stable enough that it can be stored at large pressures in the gas-phase, and can be directly seeded at large quantities in a carrier gas. From the acceptance of the Stark decelerator and from typical densities of molecular beam pulses, we expect a particle density of $\geq 10^{10}$ molecules $\mathrm{cm}^{-3}$ in the $X^{2} \Pi_{1 / 2}$, $J=1 / 2, f$ state in the cold packet. For the $\mathrm{OH}$ beam, a particle density of $10^{9}$ $\mathrm{cm}^{-3}$ appears realistic with our long Stark decelerator, operating in the $s=3$ overtone mode. With these particle densities we expect a scattering product density of about $10^{4}$ molecules $\mathrm{cm}^{-3}$. This is well within the detection limit for both the $\mathrm{OH}$ and NO radicals.

The $\mathrm{OH}$ radical can be sensitively detected using a Laser Induced Fluorescence (LIF) scheme on the strong dipole-allowed $A^{2} \Sigma^{+} \leftarrow X^{2} \Pi$ transition in the ultraviolet part of the spectrum. The NO radical can be conveniently detected by a twocolor $\left(1+1^{\prime}\right)$ Resonance Enhanced Multi Photon Ionization (REMPI) detection scheme. In this scheme, the first photon $(226 \mathrm{~nm})$ excites the NO radicals to the $A$ ${ }^{2} \Sigma^{+}$state. The second photon (either $193 \mathrm{~nm}$ or $266 \mathrm{~nm}$ ) ionizes the molecule, and the resulting ions can be detected using a standard time-of-flight mass spectrometer. Both transitions are very strong, and can be saturated with unfocussed laser beams. 
The detection laser beams can therefore be matched in size to the intersection of the molecular beams, and the inelastically scattered NO radicals are then detected with almost unity detection efficiency. This REMPI detection scheme for NO radicals is at least two orders of magnitude more sensitive than LIF detection schemes, and allows the detection of particle densities as low as $10^{2}$ molecules $\mathrm{cm}^{-3}$.

The expected signal levels in the $\mathrm{OH}+\mathrm{NO}$ scattering experiment can also be estimated from the signal levels that were obtained in the $\mathrm{OH}+\mathrm{Xe}$ scattering experiment (see section IV). In that experiment, depending on the inelastic collision channel, $0.1 \%-0.5 \%$ of the incoming $\mathrm{OH}$ radicals were inelastically scattered. From this, the density of $\mathrm{Xe}$ atoms in the target beam was estimated to be $10^{12}$ $10^{13}$ atoms $\mathrm{cm}^{-3}$. The expected density of $\mathrm{NO}$ radicals in the proposed $\mathrm{OH}+\mathrm{NO}$ experiments is two to three orders of magnitude lower. This is compensated for by the at least two orders of magnitude superior detection sensitivity of NO radicals, and by the about one order of magnitude higher density of $\mathrm{OH}$ radicals. The signalto-noise levels, therefore, are expected to be at least similar to those in the $\mathrm{OH}+\mathrm{Xe}$ experiment.

\section{The molecular synchrotron}

The combination of Stark-decelerated beams with molecular storage rings offers interesting alternatives to the crossed beam set-up for molecular scattering studies. In its simplest form a storage ring is a trap in which the particles - rather than having a minimum potential energy at a single location in space - have a minimum potential energy on a circle. As in traps, packets of molecules can be confined for times up to seconds, allowing for a large interaction time of the colliding particles. The advantage of a storage ring over a trap is that a storage ring accepts packets with a variable velocity, enabling scattering studies as a function of the collision energy. In addition, as molecules with a non-zero mean velocity can be confined in the ring, superior number densities of the molecular packets can be obtained. As in traps, the advantage of the long interaction time in storage rings is of limited use to study state-tostate inelastic collision processes. Inelastically scattered molecules in general do not experience the required potentials to stay confined in the ring. Molecular storage rings, however, are the ideal experimental arrangements to study the total (elastic + inelastic + reactive) scattering cross section, that can be inferred from the measured loss-rates of the stored molecules.

The first storage ring for neutral polar molecules was devised by bending a long electrostatic hexapole into a torus. ${ }^{44}$ A Stark-decelerated beam of deuterated ammonia molecules was decelerated to approximately $100 \mathrm{~m} \mathrm{~s}^{-1}$ and injected into the ring, in which up to 50 round trips could be observed. As a result of the longitudinal velocity spread of the packet of molecules, however, the packet gradually spreads out along the ring on making successive round trips. In the second version of a molecular storage ring, bunching elements were added to the storage ring to counteract this spreading. This storage ring consists of two hexapole half-rings that are separated by a $2 \mathrm{~mm}$ gap. By appropriately switching the voltages as the molecules pass through the gaps between the two half-rings, molecules experience a force that keeps them together longitudinally in a compact bunch. This structure is the neutral analogue of a synchrotron for charged particles. ${ }^{45}$ The synchrotron ensures that the density of stored molecules remains constant for each round trip. The broken symmetry of the synchrotron allows the injection of multiple packets into the ring, either collinear or counterpropagating, without affecting the packets that are already stored. While circling the ring, these particles can be made to interact repeatedly at well defined times and at distinct positions. The open electrode geometry allows control over experimental parameters in the interaction regions, and the introduction of electromagnetic fields and/or stationary targets in the beam path. The number of packets that can be stored simultaneously, and the efficiency of the bunching process, depend on the number of individual segments of the 
synchrotron. We are currently testing a molecular synchrotron that consists of 40 short hexapole segments.

In Fig. 6 a variety of possible experimental arrangements is shown that can be used to study collisions using a molecular synchrotron.

In Fig. 6(a) the simplest arrangement is shown to study molecular collisions in the synchrotron. The synchrotron can be loaded with multiple packets of molecules from adjacent pulses of a single injection beamline. The velocity of each packet is set by the Stark decelerator, allowing multiple trailing packets of molecules in the ring that overtake each other. Collisions between different species can be performed using the arrangements that are shown in Fig. $6(b)$ and $(c)$. The open electrode geometry of the synchrotron allows the positioning of magnetic or optical traps for molecules or atoms in the beam path. For instance, the combination of a molecular synchrotron with a sample of ultracold atoms enables the study of collisions that are relevant for sympathetic cooling schemes in molecular traps. Collisions between different molecular species are most easily performed when two separate injection beam-lines are used. In Fig. 6(c) an arrangement is shown to load multiple counterpropagating packets of molecules in the ring.

The synchrotron technology can also be exploited for detailed studies of bi-molecular state-to-state inelastic scattering in the cold $(1 \mathrm{~K}-10 \mathrm{~K})$ temperature regime. A possible experimental arrangement for this is shown in Fig. 6(d). A Stark-decelerated packet of molecules with a forward velocity in the 100 to $150 \mathrm{~m} \mathrm{~s}^{-1}$ range is bent using a quarter segment of a synchrotron. The synchrotron technology ensures that the trajectory of the packet is manipulated while keeping the molecules together in a compact bunch. A second Stark-decelerated beam is merged with the first beam, and the molecules can be made to interact in free flight some distance downstream of the exit of the bend. The velocities of both beams can be made slightly different from each other, giving access to collision energies as low as $1 \mathrm{~K}$. This experimental arrangement allows the controlled variation of the collision energy, and allows for

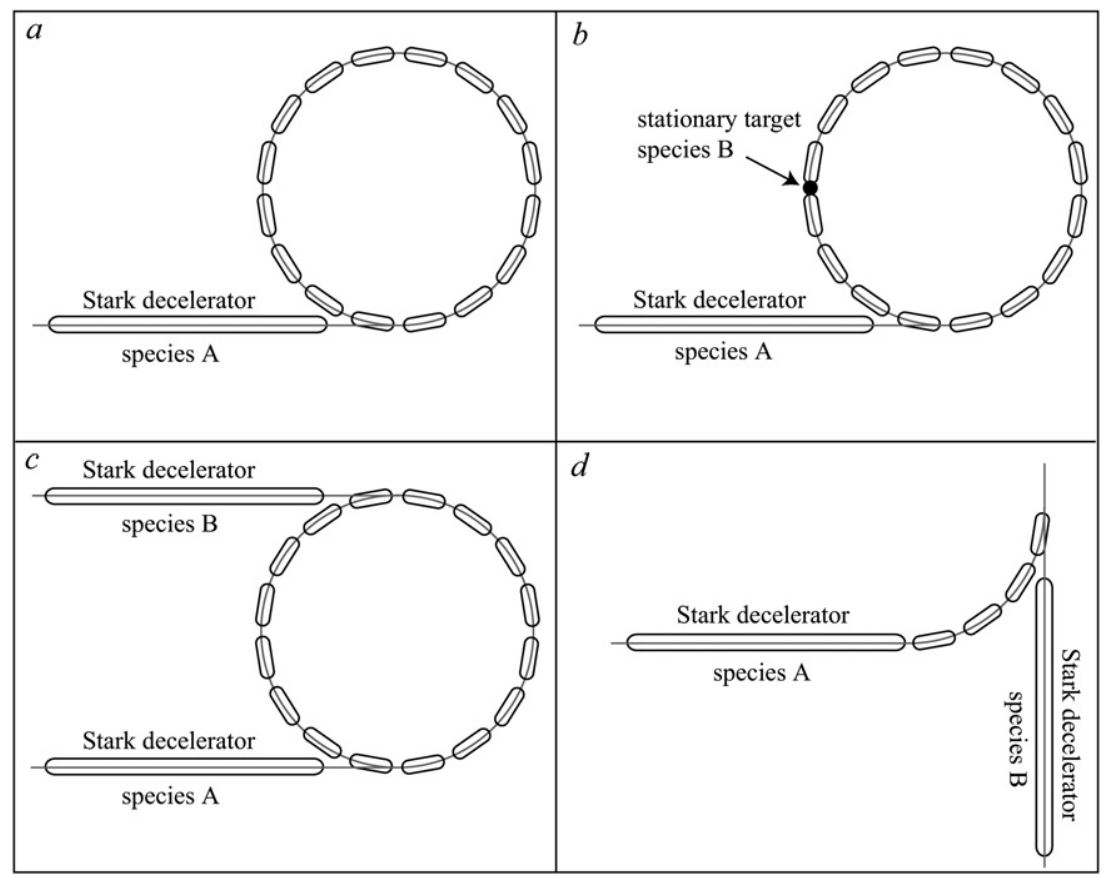

Fig. 6 Schematic presentation of four different experimental configurations that can be used to study molecular collisions, all making use of (parts of) a molecular synchrotron. 
full flexibility to control experimental parameters in the interaction region. In addition, the low collision energies are obtained with molecular beams with a relatively high forward velocity and number density; for cold collisions an interesting alternative to traps and crossed beam geometries.

\section{Molecule surface scattering}

Molecular beams with a tunable velocity also offer new possibilities in surface scattering studies. The use of directed molecular beams to probe the interaction of molecules with well-defined single crystal surfaces, is a mature field of research. Beams of state-selected molecules, in particular ground-state $\mathrm{CO}$ and $\mathrm{NO}$ molecules, are routinely used for these studies. ${ }^{46}$ After scattering from the surface, the internal rovibrational energy, the kinetic energy and the angular distribution of the scattered molecules are probed to obtain information on the molecule-surface interaction. ${ }^{47,48}$ Although the velocity of the incoming beam of molecules can normally only be changed by changing the carrier gas or the temperature of the source, the component of the velocity perpendicular to the surface - which is assumed to be the only relevant one - can be changed by simply changing the angle of incidence of the beam with the surface. It is actually an interesting question whether indeed changing the perpendicular component of the velocity by rotating the sample, for instance, has the same effect as changing the speed of the molecules under normal incidence conditions. At very low velocities of the incoming molecules, this is most likely no longer true, and the Stark decelerator offers the possibility to experimentally address this issue.

In selecting a molecule for Stark deceleration and subsequent interaction with a surface, it is important to select a molecule/surface system that has already been well-characterized. The main new feature that the Stark-decelerated beams can contribute to these studies is that beams can be produced with very low velocities, and still with a very narrow velocity spread. Obviously, the molecules that can be most efficiently decelerated, are molecules with a rather large electric dipole moment. These are not the molecules one would like to use to study the rather subtle effects at low velocities in the molecule-surface interaction, however, as these molecules typically experience a rather strong interaction with the surface.

In collaboration with Alec M. Wodtke (UCSB, Santa Barbara, CA, USA) we are setting up an experiment to study the interaction of slow beams of ground-state $\mathrm{CO}$ $\left(X^{1} \Sigma^{+}\right)$molecules with an $\mathrm{Au}(111)$ surface. In the ground-state, $\mathrm{CO}$ only has a very small dipole moment, and the interaction with the $\mathrm{Au}(111)$ surface is rather weak. To be able to decelerate $\mathrm{CO}$ molecules, they are laser-prepared in the low-field seeking component of the metastable $a^{3} \Pi_{1}, v=0, J=1$ state prior to entering the Stark decelerator using pulsed laser radiation at $206 \mathrm{~nm}$. In the metastable state, the $\mathrm{CO}$ molecules have a dipole moment of 1.5 Debye, similar to the one for $\mathrm{OH}$ and $\mathrm{ND}_{3}$, and they live long enough $(>2.6 \mathrm{~ms})$ to make it through the decelerator without significant radiation losses. In the decelerator, the metastable $\mathrm{CO}$ molecules stay together in a compact bunch, enabling their efficient transfer back to selected ro-vibrational levels in the electronic ground-state via Stimulated Emission Pumping $(\mathrm{SEP})^{49}$ at the exit of the decelerator. In this way, a fully state-selected beam of CO $\left(X^{1} \Sigma^{+}\right)$molecules in any one of the vibrational levels $\mathrm{v}^{\prime \prime}=1-6$, and always in either one of the lowest three rotational levels, can be prepared with a tunable velocity in the $10-200 \mathrm{~m} \mathrm{~s}^{-1}$ range. This slow beam will then scatter from a temperature controlled single crystal Au (111) surface, to gain information on the nature of energy transfer in molecule surface collisions in the limit of zero translational energy of incidence.

\section{Conclusions}

The Stark deceleration technique offers a new degree of control over both the internal and external degrees of freedom of polar molecules in a molecular beam. 
So far, this new molecular beam technology has been used mainly to decelerate packets of molecules to standstill, and to subsequently confine these molecules in a trap. These tamed molecular beams, however, also hold interesting prospects for scattering experiments. The exquisite level of control over molecules allows detailed molecular scattering studies as a function of the collision energy, from low to high collision energies, and with an unprecedented energy resolution. It is the challenge to design experiments that optimally exploit these new possibilities, expanding upon the rich variety of experimental methods that have been developed over decades to obtain detailed information on the molecular interaction potential. For this, we have discussed a variety of molecular systems and experimental arrangements that can be used for specific applications, ranging from crossed molecular beam set-ups to molecular synchrotrons and surface scattering machines.

The experiments that have been discussed are all challenging, but realistic. The first crossed beam experiment using a Stark-decelerated beam of $\mathrm{OH}$ radicals and a conventional beam of Xe atoms has already been successfully performed. New Stark decelerators with superior efficiency have been developed with which bimolecular scattering between two decelerated beam pulses becomes feasible. This has been discussed in more detail for the $\mathrm{OH}\left(X^{2} \Pi_{3 / 2}, J=3 / 2, f\right)+\mathrm{NO}\left(X^{2} \Pi_{1 / 2}\right.$, $J=1 / 2, f$ ) system, but, for instance, the scattering of $\mathrm{ND}_{3}$ with $\mathrm{ND}_{3}$ would experimentally most certainly be feasible as well.

The use of tamed molecular beams is of advantage in all experiments in which the velocity and/or the internal state purity of the beam are important parameters. In scattering experiments, these beams bring us one step closer to Levine and Bernstein's "ultimate scattering experiment".

\section{References}

$1 \mathrm{R}$. Levine and R. Bernstein, Molecular reaction dynamics and chemical reactivity, Oxford University Press, New York, 1987.

2 Y. T. Lee, Angew. Chem., 1987, 26, 939.

3 G. Scoles, ed., Atomic and molecular beam methods, vol. 1 \& 2, Oxford University Press, New York, NY, USA, 1988 \& 1992.

4 X. Yang, Annu. Rev. Phys. Chem., 2007, 58, 433.

5 S. Stolte, Ber. Bunsen-Ges. Phys. Chem., 1982, 86, 413.

6 D. Skouteris, D. E. Manolopoulos, W. Bian, H. J. Werner, L. H. Lai and K. Liu, Science, 1999, 286, 1713.

7 R. T. Skodje, D. Skouteris, D. E. Manolopoulos, S.-H. Lee, F. Dong and K. Liu, Phys. Rev. Lett., 2000, 85, 1206.

8 A. T. J. B. Eppink and D. H. Parker, Rev. Sci. Instrum., 1997, 68, 3477.

9 K. T. Lorenz, D. W. Chandler, J. W. Barr, W. Chen, G. L. Barnes and J. I. Cline, Science, 2001, 293, 2063.

10 X. Liu, J. J. Lin, S. Harich, G. C. Schatz and X. Yang, Science, 2000, 289, 1536.

11 S. A. Harich, D. Dai, C. C. Wang, X. Yang, S. Der Chao and R. T. Skodje, Nature, 2002, 419, 281.

12 J. J. Lin, J. Zhou, W. Shiu and K. Liu, Science, 2003, 300, 966.

13 H. L. Bethlem, G. Berden and G. Meijer, Phys. Rev. Lett., 1999, 83, 1558.

14 J. L. Bohn, A. V. Avdeenkov and M. P. Deskevish, Phys. Rev. Lett., 2002, 89, 203202.

15 N. Balakrishnan, A. Dalgarno and R. C. Forrey, J. Chem. Phys., 2000, 113(2), 621.

16 R. V. Krems, Int. Rev. Phys. Chem., 2005, 24, 99.

17 R. V. Krems, Phys. Chem. Chem. Phys., 2008, 10, 4079.

18 S. Y. T. van de Meerakker, H. L. Bethlem and G. Meijer, Nat. Phys., 2008, 4, 595.

19 A. V. Avdeenkov and J. L. Bohn, Phys. Rev., 2002, A 66(5), 052718.

20 H. L. Bethlem, G. Berden, F. M. H. Crompvoets, R. T. Jongma, A. J. A. van Roij and G. Meijer, Nature, 2000, 406, 491.

21 S. Y. T. van de Meerakker, P. H. M. Smeets, N. Vanhaecke, R. T. Jongma and G. Meijer, Phys. Rev. Lett., 2005, 94, 023004.

22 B. C. Sawyer, B. L. Lev, E. R. Hudson, B. K. Stuhl, M. Lara, J. L. Bohn and J. Ye, Phys. Rev. Lett., 2007, 98, 253002.

23 S. Hoekstra, J. J. Gilijamse, B. Sartakov, N. Vanhaecke, L. Scharfenberg, S. Y. T. van de Meerakker and G. Meijer, Phys. Rev. Lett., 2007, 98(13), 133001. 
24 L. Santos, G. V. Shlyapnikov, P. Zoller and M. Lewenstein, Phys. Rev. Lett., 2000, 85, 1791.

25 M. Baranov, L. Dobrek, K. Góral, L. Santos and M. Lewenstein, Phys. Scr., T, 2002, 102, 74.

26 J. Deiglmayr, A. Grochola, M. Repp, K. Mörtlbauer, C. Glück, J. Lange, O. Dulieu, R. Wester and M. Weidemüller, Phys. Rev. Lett., 2008, 101, 133004.

27 F. Lang, K. Winkler, C. Strauss, R. Grimm and J. H. Denschlag, Phys. Rev. Lett., 2008, 101, 133005.

28 K. K. Ni, S. Ospelkaus, M. H. G. de Miranda, A. Pe'er, B. Neyenhuis, J. J. Zirbel, S. Kotochigova, P. S. Julienne, D. S. Jin and J. Ye, Science, 2008, 322, 231.

29 J. J. Gilijamse, S. Hoekstra, S. Y. T. van de Meerakker, G. C. Groenenboom and G. Meijer, Science, 2006, 313, 1617.

30 S. Y. T. van de Meerakker, N. Vanhaecke, H. L. Bethlem and G. Meijer, Phys. Rev. A, 2006, 73(2), 023401.

31 S. Y. T. van de Meerakker, N. Vanhaecke, H. L. Bethlem and G. Meijer, Phys. Rev. A, 2005, 71(5), 053409.

32 L. Scharfenberg, H. Haak, G. Meijer and S. Y. T. van de Meerakker, Phys. Rev. A, 2009, 79, 023410 .

33 J. A. Miller and R. J. Kee, Annu. Rev. Phys. Chem., 1990, 41, 345.

34 M. C. van Beek, J. J. ter Meulen and M. H. Alexander, J. Chem. Phys., 2000, 113, 628.

35 J. J. van Leuken, F. H. W. van Amerom, J. Bulthuis, J. G. Snijders and S. Stolte, J. Phys. Chem., 1995, 99, 15573.

36 M. C. van Beek, J. J. ter Meulen and M. H. Alexander, J. Chem. Phys., 2000, 113, 637.

37 J. J. van Leuken, J. Bulthuis, S. Stolte and J. G. Snijders, Chem. Phys. Lett., 1996, $260,595$.

38 D.-C. Che, T. Matsuo, Y. Yano, L. Bonnet and T. Kasai, Phys. Chem. Chem. Phys., 2008, 10, 1419.

39 K. Sharkey, I. R. Sims, I. W. M. Smith, P. Bocherel and B. R. Rowe, J. Chem. Soc., Faraday Trans., 1994, 90, 3609.

40 A. Dehayem-Kamadjeu, O. Pirali, J. Orphal, I. Kleiner and P. Flaud, J. Mol. Spectrosc., 2005, 234, 182.

41 M. T. Nguyen, R. Sumathi, D. Sengupta and J. Peeters, Chem. Phys., 1998, 230, 1.

42 N. Vanhaecke, U. Meier, M. Andrist, B. H. Meier and F. Merkt, Phys. Rev. A, 2007, 75(3), 031402 .

43 H. L. Bethlem, F. M. H. Crompvoets, R. T. Jongma, S. Y. T. van de Meerakker and G. Meijer, Phys. Rev. A, 2002, 65(5), 053416.

44 F. M. H. Crompvoets, H. L. Bethlem, R. T. Jongma and G. Meijer, Nature, 2001, 411, 174.

45 C. E. Heiner, D. Carty, G. Meijer and H. L. Bethlem, Nat. Phys., 2007, 3, 115.

46 J. A. Barker and D. J. Auerbach, Surf. Sci. Rep., 1985, 4, 1.

47 A. M. Wodtke, J. C. Tully and D. J. Auerbach, Int. Rev. Phys. Chem., 2004, 23, 513.

48 A. W. Kleyn, Chem. Soc. Rev., 2003, 32, 87.

49 M. Silva, R. Jongma, R. W. Field and A. M. Wodtke, Annu. Rev. Phys. Chem., 2001, 52(1), 811.

50 In scattering experiments, one usually refers to fluxes rather than densities. As in most pulsed laser based detection techniques the density of the scattered molecules at a given time is recorded; we use densities here throughout. 